\title{
Corela
}

Cognition, représentation, langage

7-1 2009

Vol. $7, \mathrm{n}^{\circ} 1$

\section{Nom propre et dénomination évènementielle : quelles différences en langue et en discours?}

\author{
Laura Calabrese-Steimberg
}

\section{OpenEdition}

\section{Journals}

Édition électronique

URL : http://journals.openedition.org/corela/173

DOI : $10.4000 /$ corela. 173

ISSN : $1638-573 \mathrm{X}$

Éditeur

Cercle linguistique du Centre et de l'Ouest - CerLICO

Référence électronique

Laura Calabrese-Steimberg, « Nom propre et dénomination évènementielle : quelles différences en langue et en discours ? », Corela [En ligne], 7-1 | 2009, mis en ligne le 26 juin 2009, consulté le 01 mai 2019. URL : http://journals.openedition.org/corela/173 ; DOI : 10.4000/corela.173

Ce document a été généré automatiquement le 1 mai 2019.

\section{(c) (i) (3) (2)}

Corela - cognition, représentation, langage est mis à disposition selon les termes de la licence Creative Commons Attribution - Pas d'Utilisation Commerciale - Partage dans les Mêmes Conditions 4.0 International. 


\title{
Nom propre et dénomination évènementielle: quelles différences en langue et en discours?
}

\author{
Laura Calabrese-Steimberg
}

Nous remercions les relecteurs pour leurs remarques et commentaires.

1 Tous les jours, nous lisons dans la presse et écoutons dans les médias des expressions servant à nommer des évènements, expressions que nous mettons en circulation dans nos conversations quotidiennes et que nous reconnaissons aisément au fil du temps. La fonction référentielle de ces expressions ne nous échappe donc pas ; qu'il s'agisse d'un nom propre (Outreau) ${ }^{1}$, d'une date (le 21 avril) ou d'une expression définie (l'affaire du voile ), d'un évènement proche ou lointain, nous identifions toujours cette propriété qu'ont les désignants d'évènements (désormais $\mathrm{DE}$ ) d'opérer une référence unique, malgré la difficulté que pose le fait que l'objet désigné n'est pas une realia (mais bien une construction discursive ${ }^{2}$ ).

2 L'hypothèse qui sera soutenue dans cet article est que ces désignants ont la capacité de stocker les coordonnées de l'évènement, et en conséquence d'éveiller la mémoire des faits par la seule mention du nom. Partant de ce constat, nous voulons interroger le rapport entre la catégorie grammaticale et la mémoire des discours : dans quelle mesure leur capacité mémorielle leur permet d'opérer une référence directe, à la manière du nom propre (désormais Npr) ? Cette équivalence nom d'évènement/Npr parcourt les travaux des chercheurs qui s'intéressent à la construction de l'évènement dans les médias de masse. En effet, notre perception nous contraint souvent à interpréter ces noms comme des Npr: cet extrait d'un forum de traduction, où des traducteurs s'interrogent sur la meilleure façon d'orthographier l'expression 11 septembre (avec/sans majuscule, avec/ sans trait d'union), l'illustre :

[...] là il ne s'agit pas d'une date, il s'agit d'une locution qui devient nom propre. La révolution est un nom commun, qui ne prend donc pas de majuscule. Pourtant, quand on écrit la Révolution, on sait tout de suite de laquelle on parle. De la même 
façon, je serais très étonné qu'en pareil cas, aucune marque typographique ne vienne indiquer qu'on ne parle pas de n'importe quel 11 septembre, mais bel et bien de celui de 2001 (wordreference.com).

3 Or, bien que le fait de considérer certains $\mathrm{DE}$ comme des Npr parait tout à fait naturel ( Tienanmen, Mai-68), dans d'autres cas l'appartenance à cette catégorie présente une série de problèmes du point de vue de la description grammaticale (la crise financière, p. e.). Si certaines théories récentes pointent le rapport privilégié entre $\mathrm{Npr}$ et mémoire historique (Paveau à paraître), les DE nous confrontent à un ensemble hétérogène de noms que l'on peut difficilement ranger dans une seule catégorie.

Dans le cadre d'une Analyse du discours (désormais $\mathrm{AD}$ ) ancrée dans la description et soucieuse des formes linguistiques, nous voulons nous interroger sur la nature grammaticale et le sens discursif de ces expressions, qui font sens dans un équilibre entre langue et discours. Il s'agit de saisir un phénomène discursif à partir de son ancrage linguistique.

$5 \quad$ Nous commencerons par poser les bases d'une description discursive des DE dans le cadre du discours médiatique. Dans un deuxième temps, nous passerons en revue les différents travaux qui assimilent les $\mathrm{DE}$ au Npr, pour ensuite les confronter aux théories linguistiques du $\mathrm{Npr}^{3}$.

\section{Remarques préliminaires : de l'évènement au désignant évènementiel}

6 L'objet de notre recherche sont les noms d'évènements historico-médiatiques, ayant lieu dans l'espace public, lancés et/ou relayés par les médias de masse. Il s'agit d'une série de faits publics identifiés comme uniques, ayant acquis leur cohérence et leur notoriété grâce à un/des nom/s servant à les retracer tout au long du temps et à travers différents genres de discours. Ainsi, l'évènement est unique parce qu'il porte un identifiant, et il le porte parce qu'il est perçu comme une série unique de faits. Assumer cette circularité revient à postuler le caractère double de l'évènement historico-médiatique, factuel et discursif à la fois. Ce nom, ce « signifiant de l'évènement qui [...] atteste qu'il est lui-même un terme de l'évènement qu'il est ", comme le dit Badiou (1988: 201), est un prêt-à-dire, un élément discursif qui enregistre les coordonnées de l'évènement et, plus généralement, des discours, des images, des éléments épilinguistiques, des idées partagées et des stéréotypes qui constituent cet évènement tant qu'il a une place dans la mémoire d'une société. Nous nous référons ici à une " mémoire des mots ", telle qu'elle a été étudiée par Moirand (2007, par exemple), qui se transmet de discours en discours et non de façon interpersonnelle.

7 L'évènement historico-médiatique surgit dans l'extérieur du discours mais ne trouve son intelligibilité que dans celui-ci, et notamment dans le désignant qui vient assurer sa «traçabilité ». Ce désignant, que tout évènement doit en conséquence posséder, est le résultat d'un processus dénominatif que les médias mènent avec le consensus implicite des autres acteurs sociaux. Il est dans ce sens un fait institutionnel (Searle 1995), fruit d'un pacte dénominatif pour référer à un ensemble de faits jugé cohérent. Comme le souligne Kaufmann, «à la différence des concepts ordinaires, dont la formation est en partie basée sur l'expérience et la perception, et à la différence des concepts déférentiels scientifiques [...], les concepts sociopolitiques ne reposent que sur la communication et la 
coopération collectives » (2006: 100). Les médias donnent ainsi un nom à l'évènement, qui est censé le décrire (même dans son devenir et ses transformations) en fonction des valeurs dominantes dans une sociétét. Certains ont un haut degré de formatage (des élections, une canicule), d'autres impliquent une certaine interprétation du contexte (une crise, une guerre, des émeutes) et un petit nombre engage le positionnement public de l'énonciateur par rapport à un conflit (un génocide).

\subsection{L'évènement résulte d'un acte de baptême}

8 Ce processus de dénomination présente une énorme ressemblance avec la théorie causale du Npr proposé par le logicien Kripke, étant donné que les DE présentent un des rares cas de baptême initial dans le discours social : « un baptême initial a lieu. Dans ce cas, l'objet peut être nommé par ostension ou bien la référence peut être fixée par le biais d'une description » (Kripke, 1995 : 96, notre traduction) ${ }^{5}$. Pour Kripke, en utilisant ce nom, nous nous inscrivons dans la chaîne causale et devons donc respecter la référence initiale. La description du processus nous intéresse particulièrement dans la mesure où l'évènement n'est pas une realia mais un fait institutionnel dont nous avons connaissance par déférence, et dont la référence est fixée en partie par ostension, en partie par description. Tout $\mathrm{DE}$ a un contenu descriptif (qui n'épuise pas les caractéristiques de l'évènement mais oriente la perception que les acteurs sociaux en ont) et un contenu déictique (que nous assimilons à l'ostension), irréductible sémantiquement et qui pourrait être paraphrasé par on appelle ceci/cet évènement $X$.

9 Le désignant permet à l'évènement d'être retracé dans le discours médiatique, mais aussi dans un éventail de genres discursifs, supports et contextes : conversation quotidienne, discours scolaire ou politique, commentaires de lecteurs de la presse en ligne, blogues, forums de discussion, etc. Il a la capacité de condenser des données relatives à l'évènement, de nature plus ou moins objective (où, quoi, quand) ou subjective (images liées à l'évènement, mise en série avec d'autres évènements, discours, stéréotypes, etc.). Dans ce sens, même si les coordonnées évènementielles ne sont pas solidement établies (quand commence le conflit israélo-palestinien? Combien de faits composent le 11 septembre ? La grippe porcine est-elle vraiment d'origine porcine ?) et malgré la forte hétérogénéité qui caractérise l'évènement, le DE nous permet d'identifier une série de faits mis en série et auxquels nous assignons une cohérence, sous un nom censé lui donner une intelligibilité publique.

\subsection{Le processus de condensation d'un désignant évènementiel}

Les $\mathrm{DE}$ voient le jour dans les titres de la presse écrite, en suivant la « règle de la pyramide inversée » chère aux journalistes, selon laquelle l'essentiel de l'information doit se trouver dans le titre et le chapeau, partie saillante d'un article d'information. Ils naissent généralement dans des titres bisegmentaux à deux points (Bosredon \& Tamba 1992) sous la forme d'une expression dénominative: (Dét. quantifiant/ Ø) noyau nominal + Dét. caractérisant $t^{6}$. Le noyau du déterminant caractérisant peut à son tour être constitué par un nom commun ou un Npr (la guerre au Liban, l'affaire des caricatures, la canicule de 2003). Le premier élément (le nom déterminé) a une valeur catégorisante qui renseigne sur la nature de l'évènement, sur la classe dans laquelle il faut le ranger (guerre, affaire, 
catastrophe, etc.), tandis que le deuxième (le caractérisant) restreint son extension pour en faire le désignant d'une entité individuelle.

Dans les approches lexicales, l'élément catégorisant appartient à la catégorie des noms évènementiels, c'est-à-dire des noms qui sont compatibles avec des verbes d'évènement (se passer, survenir, se produire, avoir lieu, etc.). A la suite de Muller (2006), Fabre \& Le Draoulec (2006) distinguent des évènements forts et des évènements faibles, l'essentiel de la première catégorie étant formé de nominalisations à valeur processive (un rassemblement), mais pas uniquement (un référendum). Le deuxième groupe acquiert sa valeur évènementielle en contexte (elle est partie avant le fromage). La plupart des exemples de notre corpus appartiennent à la première catégorie, avec très peu de nominalisations, la plupart des DE présents dans le discours de l'information étant des noms strictement évènementiels ( affaire, son équivalent anglophone -gate, des noms de phénomènes atmosphériques, etc.). Pour ce qui est du deuxième groupe, certains noms s'investissent temporairement de valeur évènementielle, par exemple grippe porcine ou banlieues (voir l'exemple plus bas).

Grâce à la sémantique lexicale, nous pouvons saisir le caractère évènementiel de certains noms en langue, première étape avant de décrire le processus d'actualisation en discours. Autrement dit, si révolution est un nom générique évoquant l'idée d'un évènement, la Révolution renvoie à un évènement particulier avec des coordonnées identifiables. Le passage entre un nom évènementiel et un évènement de l'actualité se construit ainsi en discours ; il repose sur une base sémantique mais est avant tout fait de représentations, de discours et d'images épilinguistiques construits au long du temps. Notre hypothèse est que tous les $\mathrm{DE}$ sont à la base constitués d'expressions nominales comportant un nom tête évènementiel, et que même après l'effacement de ce dernier par un processus de condensation, ce qui reste du syntagme aura mémorisé les caractéristiques sémantiques du nom 7 . C'est le cas de DE tels que (la catastrophe de) Tchernobyl, (les élections du) 21 avril, (l'épidémie de) grippe porcine ou encore voile (pour l'affaire du voile ou bien pour la loi sur le voile). Ce processus de condensation de l'expression nominale, récurrent dans le discours de l'information, est à la base du phénomène de la mémoire discursive; il produit des toponymes, des dates à valeur évènementielle et des expressions définies inachevées (la crise pour la crise financière mondiale de 2008, la vache folle pour la crise de la vache folle $e^{8}$.

\subsection{Classement des DE}

Notre corpus est constitué par une série d'expressions servant à nommer des évènements historico-médiatiques, que ce soit sous leur forme originale expansée ou après le processus de condensation. Nous les avons classées d'après leur forme: expressions définies (achevées et inachevées), toponymes évènementiels, xénismes et dates en fonction évènementielle, que nous appelons héméronymes (Calabrese 2009) pour les distinguer des chrononymes (à savoir, noms de périodes historiques)9 .

Certains évènements empruntent leur nom à l'endroit où ils ont eu lieu: Tchernobyl, Outreau, Bhopal, Le Heyzel. Ce type de dénomination instaure un lien référentiel entre, d'une part, un signe qui participe déjà d'une relation référentielle, et un deuxième référent d'une toute autre nature, l'évènement. Les toponymes sont les seuls Npr purs, malgré la contamination avec le Nc qui a servi à catégoriser l'évènement, effacé par un processus d'économie linguistique et de mémorisation dans le discours social. Par ailleurs, si « les Npr purs sont perçus comme non-descriptifs ou opaques, ne renseignant 
pas sur les propriétés du particulier auquel ils sont associés " (Jonasson, 2004: 36), ils subissent toutefois d'autres processus de signifiance, que ce soit sur le plan des associations phonétiques liées à la langue de production du toponyme ou aux connaissances encyclopédiques du locuteur. Le toponyme évènementiel apporte toujours cette instruction de lecture : l'évènement en question a eu lieu à l'endroit dont il a pris le nom, ce qui permet d'actualiser la double référenciation (évènement-toponyme). Autrement dit, le toponyme évènementiel permettrait une actualisation du type un évènement a eu lieu à l'endroit dont il porte le nom, et dans ce sens il contient des éléments de description.

Les héméronymes sont des dates qui désignent des évènements : (le) 11 septembre, (le) 21 avril ou Mai 68. Comme nous l'expliquons dans Calabrese 2009, les héméronymes, comme les toponymes évènementiels, ont la capacité de bloquer toute autre référence mémorielle, contrairement à d'autres DE qui s'actualisent par rapport au contexte (la crise, la canicule, le tsunami). Ils conservent une partie des coordonnées temporelles de l'évènement, que ce soit la date ou l'année. A moins de constituer des évènements paradigmatiques (les trois DE que nous venons de citer), les héméronymes, comme les toponymes, ont besoin du contexte pour être actualisés en fonction évènementielle, comme dans l'exemple suivant :

Les deux évènements qui continuent à structurer les consciences dans ce monde arabe sont sûrement 1948 et 1967 (Courrier international du 31.5 au 6.6.07).

La circulation plus ou moins restreinte de ces DE, selon les aires géographiques, confirme leur ancrage dans la mémoire d'une société.

Leur informativité est certes faible, étant donné que le nom évènementiel est effacé (la guerre de 1967, sans parler de 1948, qui peut s'actualiser de plusieurs façons selon l'énonciateur), ce qui renforce la référenciation unique grâce à la convention de dénomination. En tant que date déterminée (par un quantifiant) ou caractérisée (par l'année), ils constituent des expressions dénominatives ; par ailleurs, leur faible contenu sémantique et leur valeur référentielle les rapprochent des Npr, au point que certains linguistes considèrent qu'elles font partie de cette catégorie (voir plus haut l'hypothèse de Flaux).

En troisième lieu, nous avons la catégorie des xénismes à valeur évènementielle ; malgré leur faible fréquence dans la presse, ils servent à nommer des faits clés de notre actualité et de notre histoire proche (le tsunami, l'intifada, la Shoah). La particularité des xénismes est d'être un nom commun dans la langue d'origine, mais leur opacité sémantique et l'association à un évènement facilitent leur comportement en tant que Npr.

19 Quant aux expressions dénominatives expansées (les DE les plus fréquents dans la presse écrite), plusieurs structures sont possibles :

(l')affaire Adidas, (l')affaire du sang contaminé, (la) guerre de Kippour, (la) guerre en Irak, etc.

20 La liste n'est pas exhaustive, mais donne une idée de la structure de ces syntagmes et de l'énorme charge sémantique qu'ils transportent. D'autres dénominations peuvent apparaître comme résultat d'une nominalisation (formes abrégées), comme dans l'exemple suivant, où banlieues résulte de l'effacement de violences (de Villiers-le-Bel) ou la crise (des banlieues) :

Banlieues : Trois cent soixante-cinq jours pour rien.

Un an après les émeutes qui ont enflammé les banlieues françaises et mis le pays en 
face de rudes réalités, rien n'a changé (Le Quotidien d'Oran repris par

Lecourrierinternational.com 27.10.06).

21 nous confronte le discours médiatique, au fonctionnement sémantico-référentiel très proche des Npr. Faut-il pour autant les ranger dans cette catégorie, malgré le fait que leur description grammaticale n'y correspond pas toujours?

\section{Nom d'évènement et nom propre}

Les récentes recherches en $\mathrm{AD}$ sur la mise en mots de l'évènement par les médias de masse font état de ce rapport entre dénominations d'évènement et Npr. Le problème se pose à deux niveaux : celui de la description grammaticale et celui de son fonctionnement en discours. Faisons un tour d'horizon des travaux qui accordent le statut de Npr aux noms servant à désigner des évènements; en termes généraux, on peut distinguer des approches plus linguistiques (dans le cadre d'une réflexion sur le Npr) et des approches plus discursives (dans le cadre d'une réflexion sur la mise en mots de l'évènement).

Bauer (1985) classe parmi les Npr les noms de phénomènes naturels (El Niño) et de faits historiques (parmi lesquels des noms d'évènements comme La Guerre de Trente Ans ${ }^{10}$ ), respectivement appelées phénonymes et praxonymes. Nous trouvons chez Leroy (2004) la définition suivante des praxonymes: "des noms propres de réalisations ou de découvertes humaines non matérielles : noms de faits historiques (la Résistance, la Guerre de Trente Ans), de maladie, de lois ou de théorèmes (la maladie d'Alzheimer, la loi de Murphy, le théorème de Thalès, le principe d'Archimède), évènements culturels (Boréalis, les Francofolies )... (34). On peut constater que les typologies référentielles du Npr ne tiennent pas compte de la morphologie des désignants (SN plus ou moins complexes, avec ou sans majuscule, mixité Npr/ Nc, etc.). En effet, la catégorie des -onymes ne fait que classer des expressions dénominatives d'après la référence (batailles, phénomènes atmosphériques, évènements), sans pour autant appartenir au Npr.

Pour sa part, Van de Velde (2003) postule l'existence de Npr de temps comme il existe des Npr de personne et de lieu (les anthroponymes et les toponymes). Les années, les noms de mois et parfois de jour sont des Npr, car «leur rôle semble bien être celui de tous les noms propres : appuyer la référence sur d'autres repères que ceux de la déixis » (Van de Velde, 2003: 40). Cette spécification de la référence leur permet de désigner des évènements particuliers, comme dans le cas de le 14 Juillet, Octobre 17, Septembre noir (catégorie désignée sous le nom de chrononymes par Büchi 1996). Malgré l'originalité de la proposition, celle-ci a sa pierre d'achoppement : elle confond les plans de la langue (noms communs de temps) et du discours (expressions servant à désigner des évènements historiques en contexte).

Les approches discursives illustrent bien l'intuition spontanée de considérer les noms d'évènements comme des Npr, mais ne s'engagent pas toujours dans une réflexion sur la catégorie (à l'exception de Veniard à paraitre). Ainsi, dans un article sur le 11 septembre publié dans Mots, le chercheur J. Frangon considère l'expression un Npr sur base du critère de la référence unique, même si elle « ne respecte pas l'ensemble des critères de ce dernier, tels que la présence d'une majuscule ou le fait que le nom propre ne puisse être traduit» (Frangon 2007 : 78), tandis que A. Krieg (Paveau, Reboul-Touré \& Lecolle, à paraître) parle de Npr d'évènements. Pour sa part, M. Veniard (à paraître) propose une 
alternative à cette étiquette problématique et appelle dénominations propres les noms de guerre, pour ne pas dissoudre la spécificité linguistique du Npr.

Confrontons maintenant ces observations aux théories du Npr. Nous examinerons des modèles purement linguistiques, comme ceux développés par Jonasson (1994), Kleiber (1996) et Bosredon (1997).

\title{
2.1 Noms propres mixtes et descriptifs
}

27 Jonasson cherche à formaliser la catégorie de $\mathrm{Npr}$ du point de vue linguistique, contrairement à la philosophie analytique qui construit son raisonnement à partir de la référence, c'est-à-dire uniquement sur le plan du discours :

\begin{abstract}
A la différence des Nc qui, en vertu de leur sens, nous aident à regrouper des objets, des individus et des phénomènes ayant des propriétés en commun, les Npr nous permettent d'isoler des entités uniques et spécifiques, en nommant des particuliers perçus à l'intérieur des catégories établies. Ils nous aident à structurer et à mémoriser un savoir spécifique à côté du savoir général systématisé par les catégories conceptuelles (1994:16).
\end{abstract}

L'auteure relie cette définition à l'approche de Kripke du désignant rigide, puisque le Npr ne doit pas être ressenti comme une description mais comme un lien stable et direct avec un objet qui peut, lui, subir des changements sans pour autant changer de nom. Ce lien, dit dénominatif, constitue selon Kleiber (1996) le sens du Npr: l'instruction de trouver dans la mémoire stable le référent qui porte le nom en question.

Par ailleurs, pour Jonasson, le signe doit pouvoir être associé à son objet en dehors de toute situation de communication. Ce lien dénominatif caractérise les Npr "purs" (anthroponymes et toponymes) et les expressions dénominatives. Jonasson appelle ces dernières Npr mixtes (la Tour Eiffel, le Collège de France) et descriptifs (La Maison blanche, l'Académie française); leur fonctionnement rappelle celui des DE par leur monoréférentialité -contrairement aux toponymes et aux anthroponymes, qui sont polyréférentiels (pour la valeur polyréférentielle du toponyme, voir Cislaru 2008). En effet, « toute expression associée dans la mémoire à long terme à un particulier en vertu d'un lien dénominatif conventionnel stable " est un Npr (ibidem: 21), à condition que l'association soit maintenue hors contexte.

Ainsi, il est légitime de considérer des expressions telles que la Révolution, la Résistance ou le Débarquement « écrites avec une majuscule comme des Npr, dans la mesure où elles sont associées dans la mémoire à un seul et même phénomène particulier » (ibidem : 26-27). Ainsi, la majuscule, bien que n'étant pas un trait définitoire, s'avère un critère discriminant pour les Npr mixtes et descriptifs ; c'est le cas aussi de certains DE, dont la majuscule fait ressortir la valeur évènementielle, comme il arrive souvent avec les héméronymes (voir l'exemple du 11-Septembre en introduction). La connaissance extralinguistique requise pour connaitre le sens de ces expressions confirme l'hypothèse qu'il s'agit de Npr. Or, le contenu sémantique de certains noms d'évènements n'est jamais effacé, comme c'est le cas pour les Npr descriptifs, notamment les géographiques (la Forêt noire) :

A la suite d'un acte de dénomination ou d'un emploi répété du Npr comme expression référentielle, ou désignateur, associé à l'entité particulière en question, un lien plus direct a pris la relève, laissant se retirer, reculer ou s'effacer le sens lexical descriptif devenu désormais superflu»; [lorsqu'ils atteignent le stade de $\mathrm{Npr}$ 《 «ils sont alors en général gardés comme dénominations, même si la réalité 
change au point qu'ils ne décrivent plus l'endroit particulier auquel ils sont associés

(Jonasson, 1994 : 127-128).

31 évènements repose largement sur leur sémantisme, qui est souvent un terrain de lutte pour les différents acteurs sociaux, dans la mesure où il instaure une interprétation de l'évènement et positionne l'énonciateur dans le débat public, comme dans les exemples suivants ${ }^{11}$ :

Le TPI confirme que les massacres de Srebrenica constituent un génocide (Lemonde.fr 19.04.04)

Le gouvernement refuse de parler de "récession" (Lemonde.fr 03.10.2008)

Or, la motivation sémantique des DE ne doit pas nous induire en erreur en ce qui concerne leur capacité descriptive : il ne suffit pas de connaître le sens du mot guerre pour identifier le référent de La guerre en Irak.

Nous voyons que les expressions définies à contenu évènementiel ne coïncident pas exactement avec les Npr mixtes ou descriptifs de Jonasson : ils établissent effectivement une relation dénominative avec une entité du monde et leur emploi exige une connaissance extralinguistique, mais elles le font via le contenu lexical et pour cette même raison ne subissent pas de désémantisation. En ce qui concerne l'obligation de garder le lien dénominatif hors contexte, ce point confronte notre corpus à un problème intéressant : si les DE gardent le lien dénominatif hors contexte (on pourrait imaginer un dictionnaire d'évènements malgré l'oubli incessant auquel les soumet l'actualité), c'est uniquement sous leur forme expansée, qui n'est pas la forme classique utilisée par la presse écrite (i. e. caricatures, banlieues, Gare du Nord), ou bien lorsqu'il s'agit de désignants paradigmatiques (le 11 septembre, contrairement à Bhopal). Leur dépendance du contexte est par ailleurs plus forte que celle des autres expressions dénominatives, et ce pour deux raisons. En premier lieu, les DE, même sous leur forme expansée, ont souvent besoin d'être désambiguïsés, ce que le contexte énonciatif (la référence au hic et nunc de l'énonciateur) fait sans trop de problème : la Guerre du Liban et la canicule sont actualisées comme celles de 2006 et 2003 respectivement pendant leurs moments discursifs (Moirand 2007) respectifs, en dehors desquels l'énonciateur doit dater l'évènement: la première guerre du Liban, la canicule de 2003. En deuxième lieu, et comme corollaire de l'aspect que nous venons d'évoquer, le désignant peut se vider partiellement de la mémoire des évènements, notamment lorsqu'il apparaît sous sa forme abrégée, comme dans le cas de la loi dite sur le voile.

Si les catégories avancées par Jonasson ne coïncident pas exactement avec le mode de fonctionnement des $\mathrm{DE}$, les critères présentés nous aident à penser notre objet et à établir la parenté des DE avec le Npr. Examinons le concept de « dénomination propre » proposé par Bosredon et repris par Veniard pour l'appliquer aux DE.

\subsection{Dénominations propres}

L'étude de Bosredon (1997) sur les titres de tableaux propose des pistes de recherche intéressantes, même s'il ignore les travaux contemporains sur le $\mathrm{Npr}^{12}$. Pour ce linguiste, les titres de tableaux remplissent deux fonctions: décrire et nommer (cette dernière fonction étant le "prédicat d'appellation", qui dans les théories du Npr équivaut au " prédicat de dénomination »), ce qui les place à mi-chemin entre le nom et la légende. En voulant souligner le lien dénominatif établi par les titres de toiles tout en gardant la 
spécificité syntaxique qui les éloigne des Npr «standards » («purs » pour Jonasson), il y voit des "dénominations ayant l'aspect de désignations", opérant un type de référenciation unique; il propose de les appeler dénominations propres. La fonction de nomination propre

est une formule prudente employée en l'absence d'une théorie du Npr couvrant toutes les formes de désignateurs d'individus. Il faut donc entendre provisoirement par cette formule toute propriété d'une chaîne linguistique qui lui permet de valider un prédicat de dénomination en tant et en tant simplement qu'il concerne le même singleton dans toutes les situations possibles où cette séquence est utilisée (Bosredon 1997 : 106) ${ }^{13}$.

Les problèmes relayés par rapport à son objet se vérifient aisément pour les DE : lien dénominatif doublé d'une fonction descriptive, monoréférencialité des expressions et syntagmes mixtes. Parenté avec le Npr donc mais forme syntaxique très différente. Pour cette raison, l'appellation est reprise par des chercheurs qui s'occupent des noms d'évènements, comme M. Veniard, qui l'emploie pour les noms de guerres :

[...] considérer l'ensemble comme un nom propre revient à nommer de la même manière le tout et la partie, ce qui ne permet pas de rendre compte de la mixité grammaticale des polylexicaux et a pour effet d'occulter une des caractéristiques spécifiques des polylexicaux : l'apparition d'un nom commun dans leur structure,

lequel apporte une dimension catégorielle au syntagme (Veniard à paraitre).

Cependant, l'appellation dénomination propre n'en est pas moins problématique : dans la mesure où tous les noms constituent des dénominations ou ont un sens dénominatif, une dénomination propre ne serait qu'un synonyme de Npr. Par ailleurs, si les expressions dénominatives peuvent assurer la référence unique, et comme l'indique Jonasson se distinguent même par cette caractéristique, il n'est pas nécessaire d'établir une fonction de nomination propre. Mais l'observation de Veniard nous rappelle l'importance de la matérialité linguistique dans l'observation de phénomènes discursifs.

D'autres modèles désignent ce type de formes linguistiques assurant la monoréférentialité entités nommantes individualisantes (Rangel Vicente 2005, dans le cadre de la praxématique) ou opérateurs d'individualisation (Pariente cité par Gary Prieur 1994 : 16), qui rassemblent les indicateurs, les Npr et les expressions définies.

\section{Conclusion}

Nous avons voulu montrer la particularité sémantico-référentielle des expressions servant à nommer des évènements, en décrivant leur fonctionnement en langue et en discours. Nous avons conclu qu'elles constituent des dénominations désignant une entité construite par le discours de l'information, en accord avec les représentations que la société se fait des évènements ayant lieu dans l'espace public. Les expressions dénominatives ont montré ne pas avoir le même degré de figement des Npr mixtes ou descriptifs, car elles reposent sur un contenu sémantique minimal. Par ailleurs, certains $\mathrm{SN}$, dans leur version condensée propre au discours de l'information, assurent avec difficulté la permanence du lien dénominatif en dehors du moment discursif correspondant (la canicule, le voile, la crise). Au contraire, les héméronymes et les toponymes évènementiels peuvent être considérés comme des Npr sans trop de difficulté, bien que le lien dénominatif doive être entretenu en permanence pour que la relation référentielle soit maintenue. C'est ainsi que certains désignants paradigmatiques nous semblent indétrônables des discours publics, tandis que d'autres évoquent avec difficulté un évènement (Bhopal, Tricastin). Dit autrement, la mémoire discursive, celle qui est logée 
dans les désignants, dépend fortement de la circulation de ceux-ci dans les discours publics. Au niveau des représentations, il est donc probable que le 11 septembre ait pour notre société un statut plus proprial que la crise financière, ce qui pourrait se vérifier par la facilité avec laquelle le premier circule hors contexte, que ce soit dans la presse de référence, des blogues politiques ou intimes, des graffiti, des affiches, des tatouages, etc.

Mais au-delà du statut grammatical des DE, ce qui ressort est la capacité de certains désignants, à savoir les toponymes évènementiels et les héméronymes, à devenir des supports privilégies de la mémoire discursive. Contrairement à La deuxième guerre mondiale, dont le sens s'appuie clairement sur une base lexicale, ou la canicule, dont la référence dépend du contexte énonciatif, des DE tels que Mai 68, le 21 avril ou Tchernobyl constituent des Npr purs, malgré la contamination sémantique qui découle d'un nom tête évènementiel (les évènements/ le mouvement de Mai 68, les élections du 21 avril, la catastrophe de Tchernobyl).

\section{BIBLIOGRAPHIE}

Badiou, A. (1988) : L'être et l'évènement, Paris, Seuil.

Bauer, G. (1985) : Namenkunde des Deutschen, Bern, Germanistische Lehrbuchsammlung.

Bosredon, B. (1997) : Les titres de tableaux. Une pragmatique de l'identification, Paris, PUF.

Bosredon, B. \& Tamba, I. (1992) : «Thème et titre de presse : les formules bisegmentales articulées par un 'deux points' ", L'Information Grammaticale 54 : 36-44.

Büchi, E. (1996) : Les Structures du « Französiches Etymologisches Wöterbuch », Recherches métalexicographiques et métalexicologiques, Tübingen, Niemeyer.

Calabrese, L. \& Rosier, L. (2005) : « Conflit d'intérêt et crise discursive : la canicule de l'été 2003 », Réseaux 100, Mons : 19-43.

Calabrese, L. (2007) : «L'émergence du contexte dans les désignants évènementiels : intuition du chercheur ou matérialité discursive ? ", Discours et contextes, Lambert Lucas : 63-71.

-(2007b) : «Quel(s) objets(s) de discours se dissimule(nt) sous la dénomination le voile?», in Cislaru G. et ali, L'acte de nommer. Une dynamique entre langue et discours, Paris, Presses Sorbonne Nouvelle : 135-148.

- (2009) : « Les héméronymes. Ces évènements qui font date, ces dates qui deviennent évènements ", Mots. Les langages du politique 88 : 115-12.

Cislaru, G. (2008) : « Le nom de pays comme outil de représentation sociale », in H. Boyer \& M.-A. PAveau (éds) Toponymes, instruments et enjeux, Mots. Les langages du politique 86 : 53-64.

Frangon, J. (2007) : «Quand le 11-septembre s'approprie le onze septembre », Violence et démocratie en Amérique Latine, Mots. Les langages du politique 85, 78-90.

Gary-Prieur, M.-N. (1994) : Grammaire du nom propre, Paris, PUF.

Jonasson, K. (1994) : Le nom propre. Constructions et interprétations, Louvain-la-Neuve, Duculot. 
Kaufmann, L. (2006) : « Les voies de la déférence. Sur la nature des concepts socio-politiques », Langage et société 117 , septembre 2006 : 89-115.

Kleiber, G. (1984) : « Dénominations et relations dénominatives », Langages, 76 : 77-94.

Kleiber, G. (1996) : « Noms propres et noms communs : un problème de dénomination », Meta : journal des traducteurs, vol. 41, $4: 567-589$. URL : http://id.erudit.org/iderudit/003323ar . Consulté le 28/01/09.

Krieg, A. (à paraître) : « A propos des 'noms propres d'évènements'. Evénementialité et discursivité », in M.-A. Paveau, S. Reboul-Touré \& M. Lecolle (éds) : « Le nom propre en discours ", Carnets du Cediscor.

Kripke, S. [1981] (1995): El nombrar y la necesidad [Naming and Necessity], trad. M. Valdés, UNAM, Mexico.

Fabre, C. \& Le Draoulec, A. (2006) : « La dimension évènementielle du syntagme nominal dans la structure avant $+\mathrm{SN} »$, Cahiers de Lexicologie 89-2 : 47-74.

Leroy, S. (2004) : Le nom propre en français, Paris, Ophrys.

Moirand, S. (2004) : « La circulation interdiscursive comme lieu de construction de domaines de mémoire par les médias ", in López-Muñoz, J.-M., Marnette, S. et Rosier, L. (éds) : Le discours rapporté dans tous ses états, Paris, l'Harmattan, p. 373-385.

- (2007) : Les discours de la presse quotidienne, Paris, PUF.

Muller, C. (1996) : La subordination en français, le schème corrélatif, Paris, Colin.

M.-A. Paveau, S. Reboul-Touré et M. Lecolle (éds.) (à paraître) : Le nom propre en discours, Carnets du Cediscor.

Rangel Vicente, M. (2005) : « La glose comme outil de désambiguIsation référentielle des noms propres », Corela, Numéros spéciaux, Le traitement lexicographique des noms propres. URL : http://edel.univ-poitiers.fr/corela/document.php?id=462 . Consulté le 10.12.2009.

Van de Velde, D. (2000) : «Existe-t-il des noms propres de temps?», in Van de Velde, D ; \& Flaux, N. (éds.), « Les noms propres, nature et détermination », Lexique 15, Presses Universitaires du Septentrion : 35-45.

Paveau, M.-A. (2008) : « Le toponyme, désignateur souple et organisateur mémoriel. L'exemple du nom de bataille ", Mots. Les langages du politique $86: 23-35$.

Searle, J. (1998 [1995]) : La construction de la réalité sociale, trad. C. Tiercelin [The Construction of Social Reality] : Gallimard.

Veniard, M. (à paraître) : « La dénomination propre la guerre d'Afghanistan en discours : une interaction entre sens et référence ", in M.-A. Paveau, S. Reboul-Touré et M. Lecolle (éds.) (à paraître) : Le nom propre en discours, Carnets du Cediscor.

\section{NOTES}

1. L'italique sera utilisé pour les noms d'évènements en mention et la typographie normale pour les noms d'évènements en usage.

2. Nous précisons que si l'évènement a une réalité physique qui a des conséquences concrètes, sa mise en mots constitue un artefact socialement construit. 
3. Les DE cités dans ce travail ont été prélevés d'un corpus de 1000 occurrences issues de la presse écrite francophone entre 2003 et 2008 ; les journaux qui le composent sont Le Monde, Libération, Le Figaro, La libre Belgique, Metro (Belgique) et Le Soir.

4. Si, comme l'explique Searle, la dénomination d'un fait institutionnel implique l'attribution d'une fonction-statut visant à agir dans l'espace public (un attentat ou une catastrophe exigent des mesures particulières), cette attribution se fait selon des protocoles d'interprétation qui suivent un patron collectif.

5. La théorie du désignateur rigide de Kripke, amplement critiquée en linguistique, s'ajuste très bien aux noms d'évènements. En effet, si elle présente de nombreux inconvénients par rapport au Npr, elle est plus adaptée aux descriptions définies proposées par les médias.

6. Pour la description grammaticale, nous suivons la terminologie de la Grammaire critique du français de Marc Wilmet (1997).

7. Ce n'est pas le lieu d'aborder la question de l'actualisation évènementielle des Npr ou des expressions définies, que nous avons discutée ailleurs (Calabrese 2007) ; rappelons cependant que le DE transporte avec lui les coordonnées de l'évènement et est capable de les actualiser par une série de marqueurs cotextuels et contextuels : position dans l'aire de la page, titre bisegmental à deux points, rapport déictique avec le contexte d'énonciation, etc.

8. La valeur évènementielle de ce syntagme, qui peut nous échapper actuellement, était évidente dans les années 1990 (voir Moirand 2004). Pour la valeur évènementielle de le voile, voir Calabrese $2007 \mathrm{~b}$.

9. Nous ne traiterons pas ici d'une autre catégorie de Npr, les noms de tempêtes et autre phénomènes météorologiques (Katrina, Wilma), car ils ne surgissent pas en discours mais sont lancés par des instituts météorologiques pour pouvoir les repérer facilement.

10. En majuscules dans la citation de Leroy ; l'original étant en allemand, il est difficile de savoir si cette majuscule est due au fait que le syntagme est interprété comme un Npr.

11. Beaucoup de combats sémantiques autour des évènements reposent sur un conflit entre sens et référence : est-ce le nom qui crée l'évènement ou vice versa ? Le cas de la canicule de l'été 2003 en France est emblématique à cet égard, les différents acteurs sociaux, y compris la presse, s'étant engagés dans le conflits de dénominations qui allaient de "catastrophe naturelle » à « crise politique », en passant par « drame estival » ou encore « controverse » ou (voir Calabrese \& Rosier, 2005).

12. Il écrit ainsi que « l'hypothèse testée dans ce travail est qu'on ne peut réduire le champ des dénominations uniques d'objets singuliers aux Npr répertoriés dans les études classiques (Pierre, Médor ou Paris). Il existe des formes remplissant un rôle référentiel à la façon des Npr mais qui ne sont pas elles-mêmes des Npr standard » (Bosredon, $1997: 4$ ).

13. Cette explication reprend la théorie du prédicat de dénomination de Kleiber 1984, par la suite abandonnée dans Kleiber 1996 et remplacée par un sens de dénomination, partagée par les Nc et les Npr.

\section{RÉSUMÉS}

Dans cet article, nous nous intéressons aux dénominations d'évènements historico-médiatiques qui circulent dans les médias, et qui ont la capacité de stocker les coordonnées de l'événement grâce à la mémoire discursive. Cette association entre la dénomination et l'événement est telle 
qu'il semble naturel de considérer ces expressions comme des noms propres, compte tenu du type de référence directe et permanente qu'ils opèrent. Or il semble que les désignants d'évènements ont des capacités différentes pour stocker la mémoire de l'actualité, selon qu'il s'agit de toponymes, de dates ou d'expressions définies. S'il semble naturel de considérer Tiananmen ou le 21 avril comme des noms propres, la grippe mexicaine ou l'affaire du voile ne fonctionnent comme des noms propres que sur le moyen terme, étant donné leur plus faible capacité mémorielle. Partant de ce constat, il nous semble important de passer d'abord par une description grammaticale de ces objets, pour ensuite interroger leur rapport à la mémoire.

In this paper we approach the question of event names in the media, which have the capacity of stocking information about those events thanks to discursive memory. The kind of bond linking the name and the event allows us to consider those expressions as proper nouns, taking into account the direct and permanent reference they establish. But name events seem to have different stocking capacities, depending on whether they are toponymes, dates or definite expressions. As some of them can be easily considered proper names (Tiananmen, 9-11), others work as proper names only during the midterm of news coverage (Mexican flu), due to their weak stocking capacity. Following those remarks, we propose that it is important to start with a grammar description of those expressions, to explore afterwards their relation to memory.

\section{INDEX}

Mots-clés : nom propre, désignants évènementiels, sémantique discursive, sémantique lexicale Keywords : proper name, event names, discursive semantics, lexical semantics

\section{AUTEUR}

\section{LAURA CALABRESE-STEIMBERG}

ULB - Ladisco 